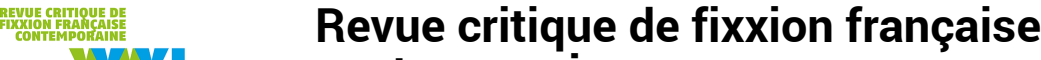 \\ XXI contemporaine
}

$21 \mid 2020$

Fictions et pouvoirs

\section{La possibilité d'une utopie romanesque pleinement émancipatrice}

Rouge impératrice de Léonora Miano

\section{Arthur Pétin}

\section{OpenEdition}

\section{Journals}

Édition électronique

URL : https://journals.openedition.org/fixxion/476

DOI : 10.4000/fixxion.476

ISSN : 2295-9106

\section{Éditeur}

Ghent University

\section{Référence électronique}

Arthur Pétin, «La possibilité d'une utopie romanesque pleinement émancipatrice », Revue critique de fixxion française contemporaine [En ligne], 21 | 2020, mis en ligne le 15 décembre 2020, consulté le 17 février 2022. URL : http://journals.openedition.org/fixxion/476 ; DOI : https://doi.org/10.4000/fixxion. 476

Ce document a été généré automatiquement le 17 février 2022.

\section{(c) (i) (9)}

Les contenus de la Revue critique de fixxion française contemporaine sont mis à disposition selon les termes de la licence Creative Commons Attribution - Pas d'Utilisation Commerciale - Pas de Modification 4.0 International. 


\title{
La possibilité d'une utopie romanesque pleinement émancipatrice
}

Rouge impératrice de Léonora Miano

\author{
Arthur Pétin
}

1 En 2124, à Katiopa, un continent africain presque entièrement unifié et devenu prospère, le chef de l'État, Ilunga, souhaite exclure du pays les Fulasi. Ces descendants de nationalistes français, ayant quitté l'hexagone au cours du XXI ${ }^{\mathrm{e}}$ siècle sous la pression d'invasions migratoires, forment une communauté appauvrie et recluse dans une identité fantasmée, en marge de la société katiopienne. La femme dont s'éprend Ilunga, Boya, universitaire spécialiste de cette communauté dite des "Sinistrés" entend, elle, tendre la main aux jeunes Fulasi dont elle croit à la possible intégration. Au sommet de l'État, on s'inquiète de cette passion qui semble faire vaciller la fermeté du héros de la libération; il s'agit donc d'écarter au plus vite cette femme dont la complexion rouge est pour le moins suspecte.

2 Voici, en quelques mots, l'histoire que nous présente l'écrivaine franco-camerounaise Léonora Miano dans Rouge impératrice ${ }^{1}$, son dernier roman, paru en septembre 2019 aux éditions Grasset. Certes, le paysage français contemporain abonde en fictions d'anticipation dystopiques, aussi diverses dans leur forme que dans leurs soubassements idéologiques: Soumission de Michel Houellebecq, 2084 : la fin du monde de Boualem Sansal ou encore Les furtifs d'Alain Damasioº ${ }^{2}$ Cependant, si on a pu déceler au sein de fictions post-apocalyptiques des propositions utopiques ${ }^{3}$, si des utopies "en friche" peuvent s'inventer dans certaines dystopies libérales de la science-fiction française contemporaine ${ }^{4}$, il n'y pas, à notre connaissance, de romans contemporains dans le champ de la littérature d'expression française qui se donnent à lire comme des utopies $^{5}$. En effet, comme l'a souligné le philosophe Miguel Abensour, depuis qu'elle a été décriée comme matrice des régimes totalitaires par la critique libérale, et caricaturée dans la pensée marxiste comme doux rêve idéaliste d'une société meilleure, l'utopie a aujourd'hui mauvaise presse ${ }^{6}$. Le roman de Léonora Miano détonne donc dans 
ce paysage littéraire et philosophique, en proposant une fiction d'anticipation utopique qui, semble-t-il, renoue le lien indéfectible, analysé par Abensour, entre utopie et désir d'émancipation. Rouge impératrice permettrait ainsi de repenser le statut, les modalités et l'efficace de ce qu'on a appelé "l'utopie narrative", en proposant une utopie pleinement romanesque qui, dégagée de toute polarisation occidentale, s'emploie à subvertir les systèmes de pouvoir, leurs discours et les imaginaires qu'ils véhiculent.

3 Si le roman présente de façon dynamique et crédible un État fédéral subsaharien prospère, fondé sur un projet civilisationnel émancipé de toute domination occidentale, il permet aussi, à la faveur d'un audacieux renversement, d'interroger à nouveaux frais les tensions identitaires de la société française actuelle. Rouge impératrice constitue, en outre, une "utopie émancipatrice" ${ }^{8}$, en ce qu'il subvertit la langue française pour l'ouvrir à un imaginaire panafricain décolonial.

\section{L'élaboration fictionnelle d'une utopie désoccidentalisée}

Rouge impératrice reconfigure les modalités de l'utopie narrative classique. Depuis Utopia de Thomas More, paru en 1516, le terme s'est imposé pour désigner le récit détaillé d'une communauté politique idéale, fondée sur un modèle d'organisation sociale que l'auteur s'attache à restituer dans son fonctionnement concret. Ancrée dans un lieu qui n'existe pas, un ailleurs défini soit dans l'espace ${ }^{9}$, soit dans le temps ${ }^{10}$, et rapidement codifiée par la structure du voyage imaginaire, l'utopie permet à ses auteurs de proposer de nouvelles conceptions de la vie collective et de dresser, en regard, une critique des normes politiques et sociales de leur temps. Toutefois, comme l'a noté Raymond Trousson, la nature essentiellement descriptive de l'utopie circonscrit toujours le récit à une exposition didactique. Et si, du XVII ${ }^{e}$ au XIXe siècle, le voyage qui conduit à l'utopie se fait plus précis, abonde en péripéties, et que le visiteur candide gagne en épaisseur fictionnelle, "tout romanesque s'évanouit sitôt qu'on pénètre en Utopie"11. Il faut attendre le début du $\mathrm{XX}^{\text {e }}$ siècle pour que le genre investisse pleinement la matière romanesque, mais c'est à la faveur d'une inversion du procédé : à "l'utopie normative" succède "l'anti-utopie", le régime politique décrit y fait figure de repoussoir et son invention vaut comme avertissement quant aux dérives du monde actuel. Si Raymond Trousson en conclut que "l'utopie ne devient roman que lorsqu'elle cesse d'être utopie" ${ }_{12}$, c'est précisément la réconciliation de ces deux ambitions narratives que permet d'envisager le roman de Léonora Miano.

5 À rebours d'un lieu utopique qui serait "celui de la suspension de l'action, de la disparition de tout rebondissement, de toute péripétie"13, Rouge impératrice se présente comme un grand roman d'amour au cœur du pouvoir, le récit de la rencontre entre le chef d'État d'une puissante fédération subsaharienne et une universitaire, spécialiste d'une communauté, les Sinistrés, qui refuse de s'intégrer dans l'État fédéral récemment constitué. Roman-fleuve, il explore les bouleversements que cet amour provoque, tant dans le quotidien du couple que dans la vision politique du monde qui s'y construit. Ilunga étant le "mokonzi" du "Katiopa unifié", la rencontre avec son âme sœur ne manque pas d'avoir des conséquences quant à l'exercice du pouvoir. Le roman orchestre ainsi les différents obstacles qui viennent s'opposer à leur union: les menées de l'épouse officielle du mokonzi, Seshamani, qui refuse que son statut soit remis en question; les conspirations du "kalala", Igazi, chef d'État-major et responsable de la 
Sécurité intérieure qui, persuadé de l'influence néfaste de Boya sur Ilunga, fomente l'assassinat de la femme rouge. C'est donc plongé au cœur de cette intrigue romanticopolitique que le lecteur, par le biais de l'alternance des points de vue des personnages, découvre le Katiopa unifié. La succession des chapitres en focalisation interne permet à Léonora Miano de se prémunir de toute description didactique du régime politique inventé : ses rouages et ses fondements sont présentés au lecteur progressivement, de façon dynamique, au gré des pensées des personnages et des multiples rebondissements qui jalonnent l'intrigue.

6 L'utopie romanesque se double, dès lors, d'une dimension pragmatique, car elle n'hésite pas à explorer le quotidien du chef de l'État fictionnel proposé. En effet, l'avènement du Katiopa est récent, et la fédération subsaharienne, en cours d'unification. Le lecteur partage ainsi les réflexions d'Ilunga qui, conscient de la fragilité de l'édifice, préfère l'approfondissement à l'élargissement, et choisit de rejeter toute stratégie de conquête militaire. Le chef d'État veut, par là même, rendre l'intégration "désirable, voire incontournable" (RI 84) auprès des régions qui se prévalent d'une singularité trop forte pour rejoindre le Katiopa unifié. De même, lorsqu'Ilunga est en route vers le Nyerere Hall où siègent les institutions (RI 276-278), l'explicitation des pensées du mokonzi qui, comme tout un chacun, songe à la journée qui l'attend, donne au lecteur le plaisir d'expérimenter par substitution l'exercice du pouvoir politique, tout en permettant à Léonora Miano de détailler certaines lignes politiques directrices : unification monétaire (on apprend qu'a été mise en place une monnaie fédérale, le pesa), maintien d'une autarcie quasi-complète (Ilunga entend être ferme avec un Président qui menace de mettre fin aux relations académiques et commerciales avec le Katiopa) et politique d'influence à l'étranger (le mokonzi décide de financer la campagne d'un membre de "l'Alliance" ${ }^{14}$ qui, à la tête d'un parti politique, brigue désormais le poste de Premier ministre d'un État occidental). Cette prise en charge, par la fiction utopique, des modalités concrètes de l'exercice du pouvoir, ne manque donc pas d'accroître sensiblement la vraisemblance du régime politique proposé.

7 Surtout, l'exposition dynamique des principes qui régissent le Katiopa unifié dessine les contours d'un régime qui, s'il retrouve quelques traits propres aux utopies narratives classiques, s'écarte des modèles politiques occidentaux. Comment ne pas noter que les textes qui, depuis le $\mathrm{XVI}^{\mathrm{e}}$ siècle, se présentent comme des utopies narratives sont tous issus de la sphère occidentale ? Rouge impératrice se singularise donc, au regard de cette histoire propre à la littérature utopique, en proposant une utopie fictionnelle désoccidentalisée, fondée sur l'émancipation vis-à-vis des anciennes puissances coloniales. Cet affranchissement se comprend d'autant mieux que la pensée utopique a pu être instrumentalisée pour légitimer la colonisation ${ }^{15}$. À la faveur d'un souvenir d'Ilunga, qui repense à sa première adresse aux gouvernants du monde, le lecteur apprend que la création du Katiopa unifié a impliqué une rupture complète avec le reste de la communauté internationale, une période de stricte autarcie. Seules les populations afrodiasporiques ont été invitées à rejoindre le continent avant la fermeture définitive des frontières. Léonora Miano assume ainsi d'aller à rebours de l'idéologie libérale de circulation des personnes et des biens qu'implique la mondialisation. Si elle retrouve, de ce point de vue, l'une des caractéristiques de l'utopie narrative classique, fondée sur un isolement géographique, elle travaille surtout à renouveler les assises théoriques du protectionnisme : dégagé de tout repli sur soi identitaire et belliciste, il est présenté, au contraire, comme un ressort essentiel 
à l'élaboration d'une fédération réussie. Ilunga précise dans son allocation que cette rupture avec le reste du monde n'a rien d'une déclaration de guerre, puisqu'il s'agit de "restituer au Continent sa vision des choses et son rythme propre" (RI 92) afin de pouvoir, ensuite, contribuer à la marche du monde. La mise en place de ce "protectionnisme quasi intégral" (ibid.) apparaît surtout nécessaire pour contraindre les différentes provinces du nouvel État fédéral à sortir de leurs dépendances à l'égard des puissances étrangères, développer des "synergies internes" (RI 90), et rendre ainsi effective l'autonomie du Continent.

La romancière prête, en outre, une attention toute particulière à certains détails du quotidien des habitants de Katiopa, concrétisant ainsi l'idéologie d'émancipation radicale de l'Occident véhiculée par le régime panafricain. Le Katiopa abolit, en effet, le calendrier occidental hérité du christianisme pour rétablir une chronologie ancestrale. Le roman s'ouvre à la "veille du San Kura 6361" $(R I 12)^{16}$, qui correspond, le lecteur ne l'apprendra qu'un peu plus loin, à l'année 2124. La portée décisive de cette rupture symbolique est ouvertement pointée dans le texte : "San Kura signifiait qu'en ce qui concernait ses choix fondamentaux, Katiopa serait seul juge” (RI 40). L'État fédéral n'est pas pour autant épargné par les inégalités sociales, ce qui permet à Léonora Miano, là aussi, de s'écarter des valeurs occidentales en dessinant les contours d'un système de redistribution des richesses. Alors qu'il suit un agent d'Igazi en mission dans une ville du sud du Katiopa, le lecteur apprend que la propriété terrienne a été abolie : pour continuer à habiter leurs résidences cossues, les classes aisées paient "un droit d'occupation", dont les sommes, "affectées à la réhabilitation de zones défavorisées,


non pas sur une logique d'égalité, concept présenté comme exogène au continent


sociétés de Katiopa n'avaient pas souvent promu l'égalité, bien au contraire, et nul ne la


qu'elle prenait en charge ceux qui devaient l'être, on ne lui reprochait pas de mener grand train" (RI 263). Si l'une des grandes caractéristiques de l'utopie narrative est la fin de la propriété privée, Léonora Miano fait, elle, de l'abolition de la propriété terrienne un régime d'obligations communautaires imposées aux classes aisées, qui garantit, par là même, une forme de paix sociale.

Le pas de côté proposé par Léonora Miano se retrouve, enfin, dans les institutions du Katiopa unifié. Le lecteur découvre en effet rapidement que le régime fédéral se structure en trois pôles : le mokonzi est à la tête d'un gouvernement au sein duquel on compte divers ministères ainsi qu'un porte-parole, responsable devant une Assemblée formée par les gouverneurs fédéraux, les "mikalayi". Plus surprenant pour un lecteur occidental, ce gouvernement agit aussi sous le contrôle du "Conseil", composé de neufs "traditionalistes éclairés" (RI 32) et présidé par une femme. Assurés de la part spirituel du régime, ils sont chargés de revitaliser certaines pratiques ancestrales en les adaptant au monde actuel. Le lecteur découvre ainsi, lors d'une réunion du Conseil avec le chef de l'État, que ses membres "ne siègent pas sous leur apparence diurne" (RI 114) mais font corps avec les éléments naturels qui leur sont à chacun associés, qu'il s'agisse d'un feu, d'une "mare luisant sous la lune" (RI 117) ou d'un palmier sauvage. La lecture potentiellement exotisante du folklore africain semble ici habilement minée par la romancière, qui souligne la nécessité stratégique de s'entretenir dans un langage 
inaccessible aux non-initiés, afin de protéger la confidentialité des délibérations du Conseil.

10 En sus de cette place centrale accordée à la spiritualité dans le fonctionnement du Katiopa unifié, Léonora Miano s'écarte du modèle politique occidental dominant, ainsi que du régime d'association propre à l'utopie narrative, en proposant un État fédéral qui, au vu de ses institutions, n'est en rien une démocratie. Seuls les membres de l'Alliance ou du Conseil délibèrent et votent pour décider des grandes orientations du régime. Or ces membres se sont autorisés d'eux-mêmes, renouvelés par cooptation au fil des générations à mesure que le projet porté par l'Alliance s'est réalisée ${ }^{17}$. Certes, la romancière insiste : il est crucial pour l'Alliance que la population adhère à son projet civilisationnel, et que cette vision soit structurée par un pacifisme intérieur. Néanmoins, il n'y a, au sein de Katiopa, ni espace public de délibération, ni mouvements politiques se réclamant d'une vision différente de celle de l'Alliance et pouvant prétendre au pouvoir. De l'aveu même d'Ilunga, Katiopa fonctionne selon "un type de démocratie devenu inhabituel, distant des méthodes auxquelles s'étaient accoutumées les nations coloniales" (RI 518). Ce caractère non-démocratique vient complexifier le statut utopique du Katiopa unifié, dont les bienfaits ne vont pas sans heurter l'un des critères fondamentaux de tout régime politique jugé légitime dans l'Occident contemporain. Il semble, à ce titre, révélateur que, parmi les chercheurs et critiques ayant fait l'éloge de Rouge impératrice dans les médias, aucun n'ait fait mention de ce point, certains allant même jusqu'à spontanément présenter le régime katiopien comme une "fédération démocratique"18.

\section{Interroger les crispations identitaires de la société française : la fiction utopique comme miroir critique}

11 C'est le propre de l'utopie narrative que de mettre en lumière, par contraste, les dérives de la société dans laquelle elle s'inscrit. Rouge impératrice tire sa force critique d'un renversement: les tenants de la théorie raciste dite du "grand remplacement" ${ }^{19}$, qui alertent contre la mort de la civilisation française sous les coups de prétendues invasions de populations immigrées venues d'Afrique, se retrouvent en situation de minorité et de précarité au sein de l'État fédéral subsaharien imaginé par Léonora Miano.

Les Sinistrés occupent une place importante dans l'intrigue du roman. Universitaire étudiant cette communauté, Boya s'est rapprochée de la matriarche de l'une des familles fulasi, Charlotte Du Pluvinage. Elle apprend ainsi que le descendant mâle des Du Pluvinage, Amaury, s'est enfui avec une chanteuse katiopienne à succès, Mawena, plongeant la famille sinistrée dans un profond désarroi. Le jeune homme, soupçonné par Igazi de vouloir infiltrer la communauté katiopienne pour reconquérir le pouvoir, est agressé et empoisonné par l'un des agents du mikalayi, de sorte qu'à son réveil, amnésique, il ne s'exprime plus qu'en langue katiopienne. Mawena, par l'entremise de l'universitaire, finit par retrouver son amant qui, auprès d'elle, recouvre peu à peu la mémoire. Ils partiront finalement rejoindre la famille de la jeune femme, loin des Du Pluvinage.

Par cette intrigue parallèle d'un amour qui transgresse les barrières raciales, somme toute assez classique, Léonora Miano approfondit et complexifie le renversement des 
systèmes de domination qu'elle opère dans le roman. Ce geste littéraire apparaît pour le moins audacieux : il s'agit de prendre aux mots les tenants de la théorie du "grand remplacement", en imaginant ce que provoquerait la présence majoritaire de citoyens français d'ascendance non-européenne dans l'hexagone ${ }^{20}$. Les Sinistrés sont en effet


du pays ancestral" (RI 16) un peu moins d'un siècle auparavant. Estimant leur pays envahi par les populations issues du continent africain, ils ont, paradoxalement, décidé de s'y réfugier: en territoires anciennement colonisés, ils pensent vivre de façon autarcique et bénéficier du respect dû à leur ancien statut de colons. La romancière franco-camerounaise insiste, d'entrée de jeu, sur le lien entre domination politique et


identité, comme un morceau de terre sacrée qui permettrait de faire un jour renaître la patrie perdue" (ibid.). Boya s'interroge, elle, sur le séparatisme des descendants fulasi qui, pour la plupart, refusent de s'associer au reste de la population katiopienne. Tout retour au pays natal semble pourtant impossible: celui-ci s'est transformé depuis le


présentant leur physionomie" (RI 69). Léonora Miano trace ainsi les contours d'une France qui, en 2124, verrait des langues comme le soninké et la darija "disput医er㜯

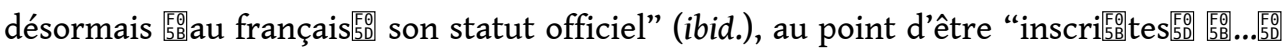
dans la Constitution" (RI 291).

Cette réalisation fictionnelle des pires craintes des suprémacistes blancs contemporains, au-delà de son caractère cocasse, permet à la romancière de dénoncer les postulats des thèses identitaires nationalistes, que sont la notion de race et le concept de supériorité culturelle. Boya interprète le communautarisme des descendants fulasi comme "une forme de psychose" (RI 72), et souligne son incompréhension devant le fait que les "Sinistrés accord $\mathbb{E D}_{\mathrm{E}}^{\mathrm{E}} \mathrm{nt}_{50}^{\mathbb{E}}$ une importance

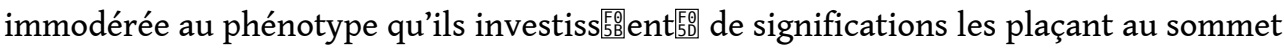
de l'espèce humaine" (RI 65). Elle remarque que c'est précisément l'invention du concept de race, cette "pathologie de l'âme" (ibid.), qui les a conduits vers le Sinistre. Le projet d'émancipation porté par l'Alliance exclut, lui, cette vision raciale. Le chef de l'État souligne ainsi que la "pensée de Katiopa avait toujours ignoré la race telle que la comprenaient ces Sinistrés, l'individu n'étant pas ici le produit de ses gènes mais celui de son environnement" (RI 17). C'est aussi aux soubassements coloniaux de l'universalisme français que Léonora Miano s'attaque, par l'intermédiaire des réflexions de Boya. Celle-ci décrit, en effet, la façon dont les Sinistrés “㗨大

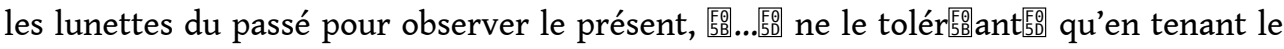
registre des avancées que leur devait l'humanité" (RI 64). Elle se souvient, par ailleurs, de la patience qu'il lui a fallu pour tolérer sans ciller les propos de Charlotte Du Pluvinage évoquant "les grandes réalisations que leur devait le genre humain et Katiopa en particulier, que l'on avait trop hâtivement tiré de la sauvagerie" (RI 71).

Si la romancière a donc parfois recours à la caricature pour dénoncer les impensés racistes de certaines idéologies nationalistes, la caractérisation des Sinistrés ne se réduit pas aux relents colonialistes exprimés par les ainés Du Pluvinage. La création par Léonora Miano de ce peuple de Sinistrés, pauvre et minoritaire dans un État subsaharien puissant, dépasse, en effet, la revanche symbolique. Au-delà de l'inversion hiérarchique entre Blancs dominants et minorités d'ascendance africaine en France, l'écrivaine engage progressivement le lecteur, par un subtil processus d'identification, à se reconnaître ou à éprouver, du moins, de l'empathie pour cette communauté en 
situation de minorité culturelle. C'est seulement par l'intermédiaire de la critique des "superstitions" (RI 25) des descendants fulasi, formulée par Ilunga, que le lecteur qui méconnait le calendrier kamite comprend que le roman se déroule en 2124 ; il est ainsi, de fait, associé à ces Sinistrés, et voit ses référents relégués au statut de simples croyances minoritaires. Ce sentiment de domination culturelle, que le lecteur d'ascendance européenne - dont fait partie l'auteur de ces lignes - éprouve peu, se prolonge lorsque Boya, écoutant Charlotte Du Pluvinage lui réciter avec émotion quelques vers de Bérénice, juge les vers de ce "poète oublié" particulièrement “monocorde医㜯” (RI 383).

Surtout, le portrait de la nouvelle génération de Sinistrés, tiraillée entre son attachement mémoriel à la patrie de ses ancêtres et son attirance pour le territoire de Katiopa où elle est née, n'est pas sans rappeler la condition propre aux Français d'ascendance subsaharienne ou caribéenne, ces "afropéens" que Léonora Miano s'est beaucoup attachée à représenter dans ses œuvres précédentes ${ }^{21}$. Boya mesure, en effet, combien la transmission par les aînés de leur ressentiment envers la population katiopienne condamne les plus jeunes Sinistrés à une situation invivable : "être de quelque part tout en ne le supportant pas, guerroyer sans fin avec une part de soimême dont il 監est㜯 impossible de se défaire" (RI 69). Cette fissure intérieure rejoint assurément le malaise identitaire de certains jeunes Français afrodescendants dans ce qui est pourtant leur pays natal.

On comprend ainsi que la relation du Katiopa unifié à la minorité sinistrée trouve de nombreux échos avec la situation hexagonale et la manière dont la France traite ses citoyens d'ascendance africaine. Pour Kabongo, l'agent d'Igazi chargé d'intercepter Amaury Du Pluvinage, la politique de "contrôles intempestifs" à laquelle on soumet sciemment les Sinistrés est une façon de "confirmer l'illégitimité de leur présence" et d'"instiller en eux un inconfort permanent" (RI 326). Le lecteur ne peut que tracer un parallèle avec les contrôles au faciès dont sont victimes, en France, les citoyens afrodescendants, et mesurer les effets discriminants et délétères que ces inspections réitérées produisent ${ }^{22}$. On saisit donc combien le discours du mokonzi aux Sinistrés, "Katiopa, tu l'aimes ou tu le quittes" (RI 411, 422, 424), dépasse le simple détournement, par ailleurs revendiqué par la romancière ${ }^{23}$, d'un slogan de l'extrême-droite française repris en 2006 par le ministre de l'Intérieur de l'époque ${ }^{24}$. Loin d'imposer la soumission pleine et entière des descendants fulasi à la culture katiopienne dominante, loin de promouvoir l'élimination de tout particularisme, la mesure décidée par le mokonzi est à comprendre comme une main tendue de l'État vers les Sinistrés, un moyen "de les reconnaître et de les placer face à leurs responsabilités" (RI 410).

Léonora Miano promeut ainsi, par la fiction, une vision ouverte et inclusive de la culture française, tout en montrant qu'un tel projet ne se fera pas sans heurts. Le lecteur est invité à observer le rejet violent que provoque chez certains personnages, tant katiopiens que fulasis, l'intégration des Sinistrés à la communauté fédérale. Si le chef d'État major considère que le "substrat commun" (RI 157) aux populations du continent africain ne peut qu'être perverti par l'assimilation de descendants européens génétiquement dominateurs et impérialistes, il rejoint, par ses conceptions essentialistes, la crainte exprimée par Monsieur Du Pluvinage, selon qui l'identité fulasie ne manquera pas de disparaitre dans ce "voyage sans retour vers la dissolution" $\left(\right.$ RI 290 ${ }^{25}$. Contre tout unanimisme factice ou angélisme benêt, Léonora Miano donne voix à des idéologies opposées par le biais de la focalisation interne, ce qui permet au 
lecteur de prendre la mesure de conceptions antagonistes de l'identité nationale. À cet égard, Rouge impératrice apparaît comme une utopie non plus monologique mais dialogique, structurée par la conflictualité des visions politiques, ce qui, selon Miguel Abensour $^{26}$, renforce précisément son potentiel émancipatoire. Lorsqu'au terme du roman, Ilunga défend les bienfaits de sa mesure devant l'Assemblée formée par les membres de l'Alliance, c'est pour mieux dénoncer la méfiance nourrie par certains katiopiens à l'égard des Sinistrés. Elle est révélatrice de leur difficulté à accepter l'idée que leur histoire a été en partie façonnée par la colonisation européenne, tout comme l'Europe l'a été par les populations africaines anciennement colonisées. Ce plaidoyer

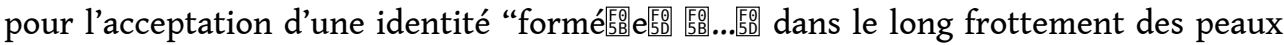
et des cultures" (RI 546) ne peut manquer, en miroir, d'interroger le lecteur quant à la situation française: qu'est-ce ce que la relégation, par la France, de ses citoyens afrodescendants hors de la "narration nationale" 27 , révèle de la conception que le pays se fait de lui-même? Rouge impératrice apparaît aussi, à cet égard, comme un geste politique, un appel à une prise de conscience, par les citoyens français d'ascendance européenne, de la "mémoire blanche" 28 que s'est inventé un pays qui se déploie pourtant sur trois océans.

\section{Les contours d'une "Afrotopie" portée par une subversion de la langue française}

Rouge impératrice constitue, enfin, et selon les termes employés par Léonora Miano, "un outil de travail" ${ }^{29}$, qui s'inscrit au sein d'un renouveau de la pensée africaine et afrodiasporique, visant à repenser l'avenir du continent africain à partir de lui-même, hors de toute domination occidentale ${ }^{30}$. Le roman participe ainsi de ce qui a pu apparaître comme "le grand retour de l'utopie africaine" ${ }^{31}$ sur la scène contemporaine. Promouvant la nécessité, pour les pays africains, d'élaborer "un projet politique, économique et social, partant de leur socioculture et émanant de leurs propres univers

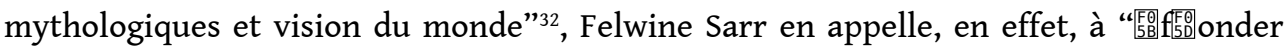


et l'imaginaire, d'ouvrir des brèches dans le présent pour permettre, depuis le réel contemporain, d'envisager de nouveaux possibles et ainsi "travailler aux conditions de

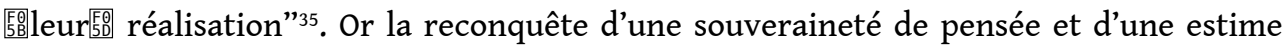
de soi apparait précisément comme le socle fondamental, précédant toute question territoriale, économique ou politique, sur lequel l'État fédéral subsaharien imaginé par Léonora Miano s'appuie pour réussir ${ }^{36}$. Les soubassements idéologiques du roman recoupent ainsi les positions défendues par l'écrivaine elle-même dans son dernier recueil d'essais et conférences, L'impératif transgressif ${ }^{37}$. La romancière donne raison à cette préséance de l'imaginaire dans la dynamique d'émancipation défendue par les courants de pensée africains contemporains, en mettant en abyme, dans la trajectoire d'établissement du Katiopa unifié, la portée décisive d'une refondation intellectuelle. Il est d'ailleurs malicieusement indiqué que la "Chimurenga conceptuelle" (RI 93) a débuté un peu plus d'un siècle avant le début du roman ${ }^{38}$. Par une forme de pensée auto-réalisatrice, Rouge impératrice proposerait donc un récit d'émancipation dans lequel les origines de l'État prospère décrit coïncideraient avec le présent même de son écriture. 
20 Ce "décentrement épistémique" 39 , valorisé par la fiction romanesque, s'y voit surtout mis en acte. Cela passe, entre autres, par la création d'une langue française renouvelée, ouverte à un imaginaire panafricain et décolonial. De nombreux termes déjà cités du roman - Katiopa, fulasi, mokonzi - sont étrangers à la langue française. Si la critique, peut-être influencée à tort par la grille de lecture du genre utopique, y a parfois vu des néologismes ${ }^{40}$, la présence d'un glossaire à la fin du roman indique qu'il s'agit bien d'emprunts à diverses langues africaines. En effet, le roman fait la part belle à ce que Rainer Grutman nomme "l'hétérolinguisme" ${ }^{41}$, soit la présence, dans un texte littéraire, d'idiomes étrangers, un procédé qui constitue, selon Jean-Marc Moura, "l'une des marques les plus caractéristiques" des "textes postcoloniaux" ${ }^{42}$. Là où des auteurs subsahariens comme Ahmadou Kourouma, Sony Labou Tansi ou, plus récemment, Patrice Nganang, ont cherché à transgresser les normes linguistiques standards du français hexagonal ${ }^{43}$, nulle perturbation de la syntaxe ni de la grammaire françaises au contact de langues subsahariennes dans Rouge impératrice. Ce respect apparent des normes classiques n'empêche cependant pas la romancière d'enrichir le lexique de la langue française tout en la subvertissant. Par un processus de défamiliarisation, qui place le lecteur dans une situation d'étrangeté par rapport à sa propre langue, Léonora Miano s'affranchit de ce que Maxime Del Fiol a très justement désigné comme les "contraintes du lectorat imposées par la structure parisiano-centrée du marché français de l'édition"44. L'écrivaine se départit ainsi de cette "esthétique du compromis" 45 que le critique pointe dans le roman La saison de l'ombre, paru en 2013.

Dans Rouge impératrice, en effet, Léonora Miano mène une entreprise de déstabilisation $\mathrm{du}$ lecteur français d'ascendance européenne, qui passe, entre autres, par le bouleversement de ses habitudes de lecture et la reconfiguration de son imaginaire. On pourrait penser que l'usage de termes issus de langues africaines favoriserait au contraire le penchant d'une part du lectorat occidental pour l'exotisme, que l'écrivaine a elle-même dénoncé ${ }^{46}$. Néanmoins, au vu de certaines réactions critiques $^{47}$, l'hétérolinguisme du texte constitue bien une source d'inconfort. Si Léonora Miano recourt déjà au glossaire dans ses trois derniers romans ${ }^{48}$, afin de traduire les quelques emprunts aux langues africaines qui émaillent les textes çà et là, l'emploi de termes étrangers au français prend, dans Rouge impératrice, une ampleur inédite. On compte près d'une centaine d'entrées dans le glossaire, qui mêle noms communs, noms propres et expressions orales. Nul signe typographique ne signale au lecteur les mots qui y sont traduits, et très peu le sont directement dans le texte par des périphrases explicatives. Surtout, de nombreux termes relatifs aux vêtements et aux parures ne sont tout bonnement pas traduits ${ }^{49}$, ce que la romancière assume de façon quelque peu taquine au début du glossaire, précisant ne pas avoir recours à la traduction de ces emprunts "lorsqu'il est aisé de les trouver à l'aide d'un moteur de recherche" (RI 603).

Léonora Miano engage donc le lecteur qui ne serait pas familier de certaines realia subsahariennes à les éclaircir par lui-même, et ce quitte à interrompre momentanément le confort de sa lecture. La création fictionnelle du Katiopa unifié permet aussi à la romancière de rendre hommage à des figures politiques marquantes de l'histoire du continent africain - qui se sont illustrées notamment par leur lutte contre l'impérialisme colonial ou leur engagement en faveur du panafricanisme - en donnant leur noms, par un jeu de toponymie semi-imaginaire, à nombre d'espaces publics. La première phrase du roman situe ainsi l'action "à quelques mètres de la place Mmanthatisi" (RI 9), régente du royaume Batlokwa durant plus de dix ans au début du 
$\mathrm{XIX}^{\mathrm{e}}$ siècle et restée dans les mémoires pour la qualité de son règne, tandis que c'est "l'avenue Ménélik II" (RI 49), empereur éthiopien qui au tournant du $\mathrm{XX}^{\mathrm{e}}$ siècle repoussa les troupes italiennes, qui mène à la résidence du chef de l'État ${ }^{50}$. Là où, dans ses précédents romans, un système de notes explicitait l'identité des figures historiques $^{51}$, aucun procédé, dans la grande majorité des cas, ne vient ici suppléer le lecteur dans son travail d'élucidation des références culturelles. Le dispositif mis en place par la romancière demande donc un effort non usuel pour le lecteur français : il lui impose, dans un roman écrit dans sa langue maternelle, d'accepter de se trouver momentanément face à des îlots d'incompréhension. Le lecteur peut, soit décider de les éclaircir aussitôt en recourant au glossaire ou, le cas échéant, à un moteur de recherche, soit, s'il préfère l'attrait d'une lecture linéaire, choisir de les laisser révéler d'eux-mêmes leur signification, en contexte et à force de récurrence. L'hétérolinguisme, d'abord subi, se fait donc peu à peu porteur de sens, constituant un seuil d'entrée pour le moins efficace en ce qu'il favorise l'immersion du lecteur dans l'imaginaire katiopien.

23 À cet égard, Léonora Miano renouvelle la langue française en l'africanisant ou, devraiton plutôt dire, en proposant un français "panafricanisé". En effet, contrairement à ce qu'elle fait dans ses précédents romans, l'écrivaine revendique, dans la note qui présente le glossaire de Rouge impératrice, de ne pas mentionner les différentes langues africaines dont sont issus les mots étrangers du texte: ces emprunts façonnent, justement, "la langue de cette histoire écrite dans l'écho de plusieurs cultures" (RI 603). À titre d'exemples, Katiopa désigne "le continent africain" en kikongo et ilunga, "l'initié" en tshiluba ; mokonzi signifie "dirigeant" en lingala et chimurenga, "rébellion" dans une autre langue bantoue, le shona. Par cette distillation continue de termes issus de diverses langues subsahariennes, Léonora Miano rend justice au caractère multilingue de l'espace culturel dans lequel vivent les francophones du sud du Sahara. Surtout, elle donne voix à la réalité panafricaine de l'État qu'elle imagine, par le biais d'une langue française enrichie, "[d]écrochée de son origine et des visées impérialistes qui lui furent assignées" ${ }^{52}$.

24 En effet, Katiopa, on l'a vu, n'est pas qu'une fédération : le régime porte une vision du monde, un projet civilisationnel qui vise à s'émanciper de toute tutelle occidentale. Or ce processus, dans le roman, passe aussi par un travail de décolonisation de la langue, à la faveur d'une dynamique de renomination. La romancière a rappelé, à plusieurs reprises, que les frontières mais aussi les noms de nombreux États subsahariens, à commencer par le terme d"'Afrique", ont été imposés par les anciennes puissances coloniales et qu'en cela ces désignations ont été, elles aussi, "des instruments d'assujettissement" ${ }^{33}$. Dès lors que l'on vise à une souveraineté subsaharienne, il lui apparaît donc "impératif" de "s'émanciper des désignations impropres" ${ }^{54}$, comme l'ont fait le Burkina Faso ou le Ghana, pour reprendre le pouvoir de s'énoncer soi-même. Dans Rouge impératrice, toutefois, cette réappropriation du pouvoir de la nomination dépasse le seul continent africain. On note en effet, dans le roman, que l'ensemble des appellations employées pour désigner des pays non occidentaux - "Katiopa" certes, mais aussi "Zhõnghuá" pour la Chine, "Bhârat" pour l'Inde ou encore "Hanguk" pour la Corée du Sud - correspondent en fait aux noms qu'emploie actuellement, dans leur langue propre, chacun de ces États pour se désigner lui-même. Ce strict refus, dans la fiction, d'en passer par le recours aux "exonymes" 55 , acte l'entreprise de désoccidentalisation de la langue française que mène la romancière. Le lecteur, de ce point de vue, prend conscience du caractère potentiellement impérialiste de 
désignations étatiques pourtant usuelles dans le monde occidental. En en faisant le vecteur d'une aspiration panafricaine et d'une visée décoloniale, Léonora Miano entreprend ainsi in fine, dans Rouge impératrice, de "katiopiser" ${ }^{6}$ la langue française.

Rouge impératrice frappe par son ambition et sa singularité dans le paysage contemporain de la littérature d'expression française, en ce qu'il met en fiction un État subsaharien fédéral et prospère, affranchi de toute ascendance occidentale, travaillé par des tensions qui ne sont pas sans évoquer la situation de la France vis-à-vis de ses populations afrodescendantes. Il n'en constitue pas moins une grande fresque amoureuse au cœur du pouvoir, portée par deux personnages africains quadragénaires en pleine puissance. Un tel constat pourrait amoindrir la portée politique du texte en l'édulcorant du côté de la romance sentimentale. Toutefois, considérant la place, pour le moins rare en sa qualité d'écrivaine subsaharienne, occupée par Léonora Miano dans le champ littéraire hexagonal ${ }^{57}$; au vu du net refus que la télévision française a récemment opposé à la romancière lorsqu'elle a proposé une fiction construite autour d'un couple d'afrodescendants non mixte ${ }^{58}$; au regard, enfin, de la faible représentation dans la littérature hexagonale contemporaine de personnages d'ascendance subsaharienne qui ne soient ni immigrés ni précaires ${ }^{59}$, on conclura qu'en sa qualité de grande fresque amoureuse, aussi, Rouge impératrice subvertit les structures de pouvoir et constitue bel et bien, quelles que soient les origines de son lecteur, une utopie romanesque pleinement émancipatrice ${ }^{60}$.

\section{NOTES DE FIN}

1. Léonora Miano, Rouge impératrice, Paris, Grasset, 2019. Nous nous y référerons désormais par l'abréviation RI.

2. Romans parus en 2015 pour les deux premiers, en 2019 pour le troisième.

3. Jean-Paul Engélibert, Fabuler la fin du monde. La puissance critique des fictions d'apocalypse, Paris, La Découverte, 2019, p. 149-170.

4. Natacha Vas-Deyres, Ces Français qui ont écrit demain. Utopie, anticipation et science-fiction au XX siècle, Paris, Honoré Champion, 2013, p. 427-433.

5. Contrairement à ce que son titre pourrait laisser penser, le roman d'Abdourahman A. Waberi, Aux États-Unis d'Afrique (2006), s'il est fondé sur un renversement des rapports de force entre Afrique et Occident, n'est pas une utopie. L'écrivain djiboutien dépeint une Afrique certes puissante mais minée par les injustices, qui n'hésite pas à user de la force pour exclure les migrants occidentaux qui fuient leurs pays dévastés. L'État fédéral africain imaginé par Waberi constitue une réplique de nos États du Nord, d'où l'africanisation cocasse de plusieurs symboles occidentaux. Il n'est pas question d'inventer une société idéale dont on expliquerait les rouages mais de construire une uchronie, afin d'interroger les logiques de domination du monde contemporain.

6. C'est à l'encontre de ces deux critiques que se place le travail de réhabilitation de l'utopie mené par Miguel Abensour, voir notamment L'utopie de Thomas More à Walter Benjamin, Paris, Sens

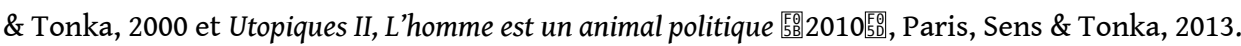


7. Jean-Michel Racault, L'utopie narrative en France et en Angleterre, 1675-1761, Oxford, The Voltaire Foundation, 1991.

8. Pour reprendre le titre de la communication de la politologue Françoise Vergès, "Utopies émancipatrices", dans Achille Mbembe et Felwine Sarr (dirs), Écrire l'Afrique-Monde, Dakar, Philippe Rey/Jimsaan, 2017, p. 243-260.

9. On pense à des textes $\mathrm{du}$ XVII ${ }^{\mathrm{e}}$ siècle comme La cité du soleil de Tommaso Campanella, La nouvelle Atlantide de Francis Bacon ou Les états et empires de la lune de Cyrano de Bergerac.

10. Louis-Sébastien Mercier écrit, en 1771, L'an 2440, rêve s'il en fut jamais.

11. Raymond Trousson, D'utopie et d'utopistes, Paris, L'Harmattan, 1998, p. 29.

12. Raymond Trousson, op. cit., p. 38. Natacha Vas-Deyres montre elle aussi, dans l'ouvrage cité plus haut, comme le tournant du XX $\mathrm{XX}^{\mathrm{e}}$ siècle, notamment la Grande Guerre, a conduit les auteurs français à délaisser les utopies statiques et déréalisantes, à la faveur de dystopies critiques, ancrées dans la science-fiction.

13. Raymond Trousson, op. cit., p. 32.

14. L'Alliance désigne, dans Rouge impératrice, l'ensemble des membres qui ont concouru à l'édification du Katiopa unifié.

15. Jean-Michel Racault, dans l'ouvrage précédemment cité, consacre un chapitre à ce lien entre entreprises de colonisation et pratique utopique, en s'appuyant sur des œuvres du début du XVIII ${ }^{e}$ siècle de Henri Duquesne et de François Leguat. Françoise Vergès, plus récemment, a montré comment au XIX ${ }^{\mathrm{e}}$ siècle, en France, la pensée abolitionniste a pu légitimer la colonisation, voir Abolir l'esclavage: une utopie coloniale. Les ambiguités d'une politique humanitaire, Paris, Albin Michel, 2001.

16. Cette chronologie correspond, en Afrique noire, au calendrier kamite.

17. On apprend, d'ailleurs, à la faveur d'une réunion du Conseil, que "la nomination de mikalayi non issus de l'Alliance” est encore prématurée, les structures de l'État devant être consolidées (RI 117).

18. On pense à l'économiste français Thomas Piketty qui, dans l'émission "L'Heure bleue" sur France inter du 19 mai 2020, loue le roman en insistant à trois reprises sur le caractère "démocratique" de la fédération inventée par Léonora Miano, URL : https://www.franceinter.fr/ emissions/l-heure-bleue/l-heure-bleue-19-mai-2020.

19. Formulée initialement par Renaud Camus en 2010, la théorie du "grand remplacement" postule que, du fait d'une immigration présentée comme massive et d'un taux de fécondité élevé, les populations d'ascendance non-européennes seraient en passe de surpasser numériquement les populations d'ascendance européennes, notamment en France, mettant ainsi en péril les prétendues traditions culturelles du pays. Cette théorie à caractère raciste et complotiste recueille toutefois aujourd'hui l'adhésion d'une proportion importante de la population française, et possède une certaine influence. Elle est reprise par des figures médiatiques, du polémiste Éric Zemmour au philosophe Alain Finkielkraut, en passant par des responsables politiques d'extrême-droite comme Marion Maréchal-Le Pen ou encore Robert Ménard. Voir à ce sujet l'article du journal Le Monde, URL: https://www.lemonde.fr/les-decodeurs/article/ 2019/03/15/la-theorie-du-grand-remplacement-de-l-ecrivain-renaud-camus-aux-attentats-ennouvelle-zelande_5436843_4355770.html, ainsi que la tribune de l'historienne et politologue Valérie Igounet, URL: https://www.lemonde.fr/idees/article/2019/03/18/attentat-dechristchurch-le-concept-de-grand-remplacement-tend-a-se-banaliser-dans-une-partie-de-lopinion-publique_5437749_3232.html, tous deux publiés le 18 mars 2019.

20. Léonora Miano dénonce elle-même le ridicule de cette idéologie et son caractère pour le moins contradictoire avec l'empire colonial français, dans son dernier recueil d'essais et conférences, L'impératif transgressif, où elle ironise sur ces "Français qui, loin d'être menacés du quart de ce que leurs aînés firent endurer aux autres, se lamentent de l'aube au crépuscule sur le 
déclin de leur civilisation, l'invasion des remplacistes, la mort de la République", Léonora Miano, L'impératif transgressif, Paris, L'Arche, 2016, p. 18-19

21. On pense aux romans Tels des astres éteints (2008) et Blues pour Élise (2010), au recueil Afropean soul et autres nouvelles (2008) et aux textes pour le théâtre, Écrits pour la parole (2012). Léonora Miano développe sa conception de l'afropéanisme dans son premier recueil de conférences, Habiter la frontière (2012). À ce sujet, voir notamment l'article de Diana Haussmann, "Afropea mise en scène : Écrits pour la parole de Léonora Miano", ainsi que celui d'Abdoulaye Imorou, "Léonora Miano en Afropéa. Invitation dans un monde qui s'invente", dans Guillaume Bridet, Virginie Brinker, Sarah Burnautzki et Xavier Garnier (dirs), Dynamiques actuelles des littératures africaines. Panafricanisme, cosmopolitisme, afropolitanisme, Paris, Karthala, 2018, p. 163-189.

22. La réalité en France de contrôles d'identité fondés "sur des caractéristiques physiques associées à une origine réelle ou supposée, sans aucune justification objective préalable", ainsi que le caractère discriminatoire et illégal de telles pratiques, ont été reconnus et sanctionnés par la Cour de cassation en novembre 2016.

23. Léonora Miano, dans un entretien, présente ce renversement comme "un pied de nez à tout cet esprit droitard", « Rencontre avec Léonora Miano : Je n'habite pas le monde en me sentant victime de l'histoire ", propos recueillis par Léonard Billot, Les inrockuptibles, n 1237, 14 août 2019, p. 17.

24. Nicolas Sarkozy déclarait le 22 avril 2006 : “S’il y en a que cela gêne d'être en France 䜿...医, qu'ils ne se gênent pas pour quitter un pays qu'ils n'aiment pas”. Philippe de Villiers, dont le parti avait alors pour slogan "La France, tu l'aimes ou tu la quittes", l'avait accusé de plagiat. Sur les origines du slogan, voir l'article de Christiane Chombeau, «La France, aimez-la ou quittez-la, bataille pour un slogan ", Le Monde, 23 mai 2016, URL : https://www.lemonde.fr/societe/article/ 2006/05/23/la-france-aimez-la-ou-quittez-la-bataille-pour-un-slogan_774922_3224.html.

25. L'obsession pour la pureté identitaire et le refuge dans un passé mythique ne sont toutefois pas l'apanage de courants nationalistes occidentaux. Ils se retrouvent aussi au sein de courants afrocentristes radicaux que Léonora Miano interroge dans Tels des astres éteints.

26. Voir, à ce propos, "L'homme est un animal politique. Entretien avec Miguel Abensour", propos recueillis par Sonia Dayan-Herzbrun, Anne Kupiec et Numa Murard, Mouvements, 2006, vol. 3-4, n²45-46, p. 75.

27. Léonora Miano, Habiter la frontière, Paris, L'Arche, 2012, p. 60.

28. Ibid.

29. C'est en ces termes que Léonora Miano présente le Katiopa unifié dans un entretien pour le magazine Jeune Afrique, "Léonora Miano: Il faut sortir du piège de la race », propos recueillis par Mabrouck Rachedi, Jeune Afrique, 25 septembre 2019, URL : https://www.jeuneafrique.com/mag/ 832297/culture/leonora-miano-il-faut-sortir-du-piege-de-la-race/.

30. On pense notamment à l'essai de l'économiste et écrivain sénégalais Felwine Sarr, Afrotopia (2016), et à l'organisation, depuis octobre 2016, des "Ateliers de la pensée" de Dakar, fondés par Felwine Sarr et le philosophe Achille Mbembe, où se réunissent chaque année intellectuels et artistes du continent et de ses diasporas pour réfléchir aux devenirs d'une Afrique en pleine transformation. Ces rencontres ont déjà donné lieu à deux ouvrages collectifs importants : Écrire l'Afrique-Monde (2017) et Politique des Temps. Imaginer les devenirs africains (2019).

31. Pour reprendre le titre de l'article de Séverine Kodjo-Grandvaux, publié sur le site du journal Le Monde, le 23 février 2020, URL : https://www.lemonde.fr/afrique/article/2020/02/23/le-grandretour-de-l-utopie-africaine_6030544_3212.html.

32. Felwine Sarr, Afrotopia, Paris, Philippe Rey, 2016, p. 25.

33. Ibid., p. 14.

34. Ibid.

35. Ibid., p. 136.

36. Le lecteur de Rouge impératrice apprend, au fur et à mesure, que la mise en place du Katiopa unifié constitue le point d'aboutissement de plusieurs luttes de libération, les "Chimurenga", 
dont la première, présentée comme la plus décisive, a consisté en un long travail de refondation intellectuelle mené par les membres de l'Alliance auprès des populations d'Afrique subsaharienne et des Afrodescendants établis dans le reste du monde. Il comprend que ce procédé de réarmement spirituel a favorisé une réhabilitation de la conscience de soi, affranchie de toute représentation négative ou honteuse façonnée par des siècles de domination occidentale.

37. Léonora Miano y souligne, en effet, que la "souveraineté intellectuelle du continent enfantera


complaisance, ni autoflagellation", L'impératif transgressif, op. cit., p. 174.

38. On le comprend notamment lorsqu'Ilunga, se référant à des chanteurs de hip-hop des années 2000, parmi lesquels le gabonais Lord Ekomy Ndong, voit en eux de nobles "combattants de la Première Chimurenga", RI, p. 126.

39. Felwine Sarr, op. cit., p. 111. C'est cette entreprise de décentrement de la pensée et des humanités, dégagées des normes de savoirs imposées par la violence coloniale, que promeuvent, entre autres, les contributeurs du volume Écrire l'Afrique-Monde. Ils soulignent, pour la plupart, l'urgence de procéder à une "décolonisation épistémique", pour reprendre l'expression de la philosophe Nadia Yala Kisukidi, dans Achille Mbembe et Felwine Sarr (dirs), op. cit., p. 56.

40. On pense, par exemple, à l'entretien filmé de la romancière pour Mediapart, publié sur le site du journal le 18 septembre 2019. Le journaliste postule que la plupart des termes étrangers sont inventés. Alors que Léonora Miano précise qu'il n'y a aucun néologisme, il insiste pour en trouver tout de même quelques uns, ce qui amène l'écrivaine à le contredire à nouveau en utilisant, cette fois-ci, un exemple. URL: https://www.mediapart.fr/journal/culture-idees/180919/leonoramiano-l-afrique-aussi-le-droit-d-incarner-l-amour.

41. Rainer Grutman, Des langues qui résonnent. L'hétérolinguisme au XIX ${ }^{e}$ siècle québécois, Québec, Fides, 1997, p. 37. Sur cette notion, voir aussi Myriam Suchet, L'imaginaire hétérolingue. Ce que nous apprennent les textes à la croisée des langues, Paris, Classiques Garnier, 2014.


89. Les textes "postcoloniaux" désignent ici les œuvres d'expression française, issues de pays ayant été marqués par la domination coloniale, qui déconstruisent les structures de domination dont elles ont hérité, à commencer par la langue des anciens colons.

43. Pour l'écrivain ivoirien Ahmadou Kourouma, chez qui le malinké ne cesse de se mêler au français via des néologismes, des emprunts, des distorsions syntaxiques ou grammaticales, voir Les soleils des indépendances (1976) ou Monnè, outrages et défis (1990). Nous pensons aussi à La vie et demie (1979) du congolais Sony Labou Tansi, et à Temps de chien (2001) du camerounais Patrice Nganang, où syntaxe et sémantique françaises se voient bousculées au contact, entre autres, du kikongo et du lingala pour le premier, et du camfranglais pour le second.

44. Maxime Del Fiol, "Réveiller la mémoire de mondes disparus. Les enjeux postcoloniaux de la recréation littéraire de l'Afrique précoloniale dans La Saison de l'ombre (2013) de Léonora Miano”, Komodo 21, n 11, 2019, URL : http://komodo21.fr/reveiller-memoire-de-mondes-disparus-enjeuxpostcoloniaux-de-recreation-litteraire-de-lafrique-precoloniale-saison-de-lombre-2013-deleonora-miano.

45. Ibid.

46. Léonora Miano déplore, en effet, que “de nombreux lecteurs occidentaux 宧...嚂 viennent aux écrits des Subsahariens dans le but de s'offrir une promenade exotique à moindre coût", L'impératif transgressif, op. cit., p. 60.

47. On pense notamment aux propos tenus dans l'émission "Le Masque et la Plume" sur France Inter, datée du 20 octobre 2019, dans laquelle certains critiques se plaignent des "vocables africains qui freinent la lecture", au point que le roman leur paraît "hermétique" et très difficile d'accès. D'autres, au contraire, s'en réjouissent, reconnaissant ne pas chercher à connaître forcément le sens des mots étrangers. Il est frappant, en tout cas, de constater que c'est bien 
cette introduction dans la langue française de termes subsahariens, qui constitue le cœur du débat sur Rouge impératrice dans l'émission. URL: https://www.franceinter.fr/emissions/lemasque-et-la-plume/le-masque-et-la-plume-20-octobre-2019.

48. On trouve un glossaire dans La saison de l'ombre (2013), Crépuscule du tourment (2016) et Crépuscule du tourment II (2017), qui compte en moyenne une trentaine d'entrées.

49. Ainsi, dès les premières pages du roman, à la faveur d'une entrevue entre Ilunga et son ministre des Affaires diasporiques, on peut lire: "Voyant le kurta cramoisi qu'avait revêtu Kabundi, le mokonzi se félicita d'avoir opté pour un agbada noir, porté sur un sokoto étroit" (RI 121). Si mokonzi et sokoto, qui signifie "pantalon étroit", sont traduits dans le glossaire, kurta, qui désigne une "chemise ample qui descend jusqu'aux genoux ou à mi-cuisse" et agbada, qui est une "longue et ample tunique", ne le sont pas.

50. Le spectre historique pris en charge par Léonora Miano est particulièrement large : du "boulevard Rei Amador" (RI 211), meneur d'une révolte des esclaves à Sao Tomé en 1595, à "l'hôpital Mukwege" (RI 329), du nom du gynécologue congolais qui a reçu le prix Nobel de la paix en 2018 ; de la "place Amina de Zaria" (RI : 489), reine guerrière célébrée pour sa bravoure, qui vécut dans la deuxième moitié du XVII e siècle au nord du Nigeria, au "Nyerere Hall" (RI 525), du nom de Julius Nyerere, premier président de la République de Tanzanie durant la deuxième moitié du XX $\mathrm{XX}^{\mathrm{e}}$ siècle et fervent défenseur du socialisme africain.

51. Ces notes, infrapaginales dans Tels des astres éteints, sont reportées à la fin du roman dans Crépuscule du tourment I et II.

52. Léonora Miano, L'impératif transgressif, op. cit., p. 98.

53. Léonora Miano, “De quoi Afrique est-il le nom ?", Achille Mbembe et Felwine Sarr (dirs), op. cit., p. 106.

54. Léonora Miano, L'impératif transgressif, op. cit., p. 174.

55. Un exonyme est le nom géographique utilisé dans une langue, pour désigner un lieu situé en dehors du territoire dont cette langue est la langue officielle.

56. Pour transposer l'expression employée par Ahmadou Kourouma, expliquant avoir "entrepris de malinkiser le français" dans son premier roman, Le soleil des indépendances. Voir "Entretien avec Ahmadou Kourouma. Je suis toujours un opposant", propos recueillis par Aliette Armel, Le Magazine littéraire, $\mathrm{n}^{\circ} 390$, septembre 2000.

57. Léonora Miano bénéficie, en effet d'un succès tant critique que public. Son œuvre, récompensée par plusieurs prix littéraires nationaux prestigieux, est publiée par une grande maison d'édition parisienne, dans sa collection de littérature française. Nous renvoyons à l'analyse proposée par la romancière elle-même de sa place singulière dans le champ littéraire hexagonal : "Sacrée marginale", L'impératif transgressif, op. cit., p. 113-139.

58. Léonora Miano évoque cette déconvenue dans L'impératif transgressif, op. cit., p. 78-80. Elle y revient aussi dans l'entretien à Mediapart évoqué plus haut.

59. La romancière s'étonne, dans une conférence donnée en 2011, de "lire des romans français contemporains, écrits par des auteurs blancs, dans lesquels on ne voit pas un seul personnage




cit., p. 79-80. Il ne nous semble pas, une dizaine d'années plus tard, qu'un tel constat soit invalidé. 60. Signalons, pour finir, que depuis l'écriture du présent article, Léonora Miano a fait paraître un essai important, Afropea. Utopie post-occidentale et post-raciste (2020), dans lequel elle développe bon nombre des perspectives que nous avons tracées ici à partir de l'étude de Rouge impératrice. À celles et ceux qui souhaiteraient approfondir les différents aspects de la pensée politique de l'écrivaine, nous en recommandons vivement la lecture. 


\section{RÉSUMÉS}

Le présent article s'attache à montrer combien Rouge impératrice, dernier roman de l'écrivaine franco-camerounaise Léonora Miano, détonne au sein de la littérature contemporaine d'expression française : il propose une fiction d'anticipation utopique qui, dégagée de toute polarisation occidentale, s'emploie à subvertir les systèmes de pouvoir actuels, leurs discours et les imaginaires qu'ils véhiculent. À travers le récit de la rencontre, en 2124, entre le chef d'État d'une puissante fédération subsaharienne et une universitaire à la complexion rouge, spécialiste d'une communauté de descendants d'immigrés français nationalistes qui refusent de s'intégrer dans l'État fédéral récemment constitué, Léonora Miano propose une utopie romanesque désoccidentalisée : la fresque amoureuse s'accompagne de l'exposition dynamique, pragmatique et crédible d'un État fédéral subsaharien prospère, fondé sur un projet civilisationnel affranchi de toutes formes de domination héritées du colonialisme. Mais la fiction utopique vaut aussi comme miroir critique : à la faveur d'un audacieux renversement, qui fait des suprémacistes blancs contemporains une minorité en situation de domination culturelle, le roman permet d'interroger à nouveaux frais les crispations identitaires de la société française actuelle. Rouge impératrice participe, en outre, d'un renouveau de la pensée africaine, qui revendique une pleine souveraineté intellectuelle dans la dynamique d'émancipation vis-à-vis des anciennes puissances coloniales ; le propos ressaisit donc enfin la façon dont le roman met en acte ce décentrement épistémique, en ouvrant notamment la langue française à un imaginaire décolonial panafricain.

\section{INDEX}

Mots-clés : littérature francophone, utopie romanesque, roman d'anticipation, fiction postcoloniale

\section{AUTEURS}

\section{ARTHUR PÉTIN}

Université Grenoble Alpes 